\title{
Rural-urban dimensions of the perception of malaria severity and practice of malaria preventive measures: insight from the 2018 Nigeria Demographic and Health Survey
}

\author{
Precious Adade Duodu ${ }^{1 *}$ (D), Veronica Millicent Dzomeku², Chiagoziem Ogazirilem Emerole ${ }^{3}$ (D), \\ Pascal Agbadi ${ }^{2}$ (D), Francis Arthur-Holmes ${ }^{4}$ and Jerry John Nutor ${ }^{5}$ \\ ${ }^{1}$ Department of Nursing and Midwifery, School of Human and Health Sciences, University of Huddersfield, \\ Huddersfield, UK, ${ }^{2}$ Department of Nursing, College of Health Sciences, Kwame Nkrumah University of Science and \\ Technology, Kumasi, Ghana, ${ }^{3}$ Department of Public Health, School of Health Technology, Federal University of \\ Technology, Owerri, Nigeria, ${ }^{4}$ Department of Sociology and Social Policy, Lingnan University, Tuen Mun, Hong Kong \\ and ${ }^{5}$ Department of Family Health Care Nursing, School of Nursing, University of California San Francisco, San \\ Francisco, CA, USA \\ *Corresponding author. Email: p.a.duodu@hud.ac.uk
}

(Received 26 February 2021; revised 30 June 2021; accepted 30 June 2021; first published online 17 September 2021)

\begin{abstract}
Morbidities and mortalities caused by malaria are still a serious issue in Nigeria, with the country accounting for $25 \%$ of malaria morbidities and $24 \%$ of malaria mortalities globally in 2018 . Treated bed nets reduce the incidence of malaria, but not all Nigerians use them. This study aimed to examine the factors associated with treated bed net usage, including perceived severity of malaria, and the rural-urban differences in the relationship between socio-demographic factors and use of treated bed nets in Nigeria. The analytic sample size comprised 40,693 women aged 15-49 years. Poisson regression and bivariable and multivariable analyses were used to test the study hypothesis that women who agreed that malaria could potentially lead to death would be more likely to adopt malaria preventive measures, including treated bed net use. About $48 \%$ of the women slept under a treated mosquito net the night before the survey. Those who perceived that malaria could lead to death had a higher likelihood of using a treated bed net in the urban, rural and combined samples. However, in the multivariable model, the association between perceived malaria severity and use of a treated bed net was only significant for rural women $(A P R=0.964,95 \%$ CI: $0.933,0.996)$. The results unexpectedly suggest that rural Nigerian women who perceive malaria to be severe have a lower likelihood of using treated bed nets. Also, rural-urban variations in the relationship between the socio-demographic variables and use of treated bed nets were observed. Policies should consider the observed rural-urban dichotomy in the influence of perceived severity of malaria and other socio-demographic factors on women's use of treated bed nets in Nigeria.
\end{abstract}

Keywords: Treated nets; Social determinants; Malaria incidence

\section{Introduction}

Malaria, a vector-borne disease, is a serious public health concern and continues to be a major focus of the Sustainable Development Goals (Dawaki et al., 2016; Okunlola \& Oyeyemi, 2019). In 2019, about 229 million cases and 409,000 malaria-associated deaths occurred worldwide, with nearly half of the world's population being at risk (World Health Organization, 2020). Although about 97 countries and territories in the tropics and sub-tropics are burdened by malaria, fifteen 
contributed the highest burden with $80 \%$ of malaria-associated deaths (Okunlola \& Oyeyemi, 2019). Regionally, sub-Saharan Africa (SSA) is accountable for about $92-94 \%$ of the global malaria burden (Okunlola \& Oyeyemi, 2019; World Health Organization, 2020).

Since 2010, there has been a global reduction in the rate of decrease in malaria incidence. The decrease generally halted in 2014, and reversed in some countries (World Health Organization, 2018). In Nigeria, about $100 \%$ of the population is at risk of malaria, and the country contributed the highest proportion of malaria cases (25\%) and malaria deaths (24\%) globally in 2018 (World Health Organization, 2019b). It was also one of two countries with the highest rates of malaria infection in pregnancy (World Health Organization, 2019b). Nationally, malaria contributes about $60 \%$ of all outpatient visits to health facilities and $30 \%$ of child mortality (Dawaki et al., 2016; Andrada et al., 2019).

In 2019, an estimated US\$3 billion was spent on malaria control and elimination globally, with governments of endemic countries contributing about US\$900 million (31\%) (World Health Organization, 2020). Malaria has been shown to intensify poverty, limit educational opportunities and increase absenteeism in schools and at work in affected populations (Van DammeOstapowicz et al., 2012; Ghahremani et al., 2014). However, despite a scaling up of efforts, global malaria reduction and long-term elimination programmes have not seen much success due to lack of resources, economic constraints, long distance to health facilities, limited knowledge of malaria, parasitic drug resistance and insecticide-resistant mosquitoes (Pell et al., 2011; Ghahremani et al., 2014; Diiro et al., 2016).

Very importantly, the World Health Organization (WHO) devised the Global Technical Strategy for Malaria 2016-2030, which called for the elimination of malaria in at least ten countries that had the greatest burden of the disease in 2015 by the year 2020. In 2016, 21 countries spanning five regions were identified by the WHO as being able to defeat malaria by 2020 , based on the likelihood of elimination across key criteria, and these countries formed the E-2020 initiative. Recently, WHO launched the E-2025 initiative, which aims to halt transmission of the disease in 25 more countries by 2025 (World Health Organization, 2019a, 2021). Measures such as controlling mosquito breeding sites and the use of long-lasting insecticide-treated nets (LLINs) play key roles in achieving these targets (World Health Organization, 2020). Sadly, only $40 \%$ of the at-risk population worldwide slept under LLINs in 2018 (World Health Organization, 2019b). The Nigerian Government, through the National Malaria Elimination Programme (NMEP) and collaborators, have instituted malaria prevention and treatment interventions towards achieving the 2014-2020 National Malaria Strategic Plan (NMSP) (Federal Ministry of Health, 2014). This strategic plan aimed to reduce the incidence rate of malaria to less than 5000 per 100,000 persons and reduce deaths attributable to malaria to zero by 2020 . One of the objectives of this goal was to provide at least $80 \%$ of the targeted population with appropriate preventive measures by 2020, including increased LLIN ownership coverage (Federal Ministry of Health, 2014; Andrada et al., 2019). A mixed-model approach, including free mass LLIN distribution campaigns (e.g. through immunization and antenatal care services) was devised by the NMEP (Andrada et al., 2019). Nigeria's NMEP resulted in LLIN use increasing from $8 \%$ in 2008 to $61 \%$ in 2018 (Ameyaw et al., 2020).

In Nigeria, socio-demographic and economic factors play key roles in the uptake of malaria prevention and control measures. These vary by region, place of residence, education and other maternal demographic characteristics (Ameyaw et al., 2020). Malaria is reported to mainly affect vulnerable communities (Gómez et al., 2017). Young children are biologically vulnerable to malaria. Also, less educated people and housewives living in precarious conditions are at high risk of malaria infection (Gómez et al., 2017). Contrasting studies have indicated that formal education is both a positive (Dike et al., 2006; Diiro et al., 2016) and negative (Ahmed \& Zerihun, 2010; Auta, 2012) predictor of uptake of malaria prevention and treatment strategies. The majority of malaria cases are thought to occur in rural areas worldwide, but with a growing number of urban and peri-urban cases (Padilla et al., 2015). Rapid rural-urban migration has resulted in 
populations living in poor socioeconomic conditions with inadequate housing infrastructure and a lack of public services. These settlements usually have inadequate sanitation and poor surface water drainage, leading to the development of water bodies and vegetation-rich areas, which act as breeding grounds for mosquitoes, especially Plasmodium falciparum and Plasmodium vivax (De Silva \& Marshall, 2012; Padilla et al., 2015). In Nigeria, the human malaria parasites include $P$. falciparum, $P$. ovale and $P$. malariae, among others. Plasmodium falciparum, transmitted through the bites of competent female mosquitoes of the genus Anopheles, is said to account for around $90-95 \%$ of malaria infections, while $P$. malariae and $P$. ovale are estimated to be responsible for less than 5\% of infections (Oduola et al., 2013; World Health Organization, 2018).

Oduola et al. (2013) assessed malaria vector abundance and diversity in rural and urban communities in three selected major towns in south-western Nigeria, to determine the impact of urbanization on the diversity and abundance of the Anopheles species associated with malaria transmission in human habitations. Ten Anopheles species were identified in the rural communities, with only four species, namely A. gambiae, A. funestus, A. moucheti and A. nili, occurring in more than $50 \%$ of the rural communities. On the other hand, eight Anopheles species were identified in the urban communities, with only one species (A. gambiae) occurring in all urban communities, while three major vectors (A. funestus, $A$. moucheti and $A$. nili) occurred in no more than $20 \%$ of the urban communities. Additionally, some mosquito species seem to easily adapt to new breeding conditions in urban settings (De Silva \& Marshall, 2012; Oduola et al., 2013). Another study in Malawi reported that Anopheles abundance was greater during rainy seasons, with $A$. funestus being more abundant than A. arabiensis, although the female of both species significantly increased with distance from the urban centre (Dear et al., 2018).

These socio-demographic and economic factors and 'social characteristics within which living takes place' serve as structural mechanisms that affect a wide range of health conditions (World Health Organization, 2010; Committee on Educating Health Professionals to Address the Social Determinants of Health et al., 2016). These factors stratify populations into groups, which results in health inequalities, including in malaria transmission, and invariably shapes specific determinants of health status and reflects individuals' experiences in exposure and vulnerability to health-compromising conditions (World Health Organization, 2010). Together with contextual factors and the socioeconomic position of individuals from such stratification, these underpin the Social Determinants of Health (SDH) framework (World Health Organization, 2010; Committee on Educating Health Professionals to Address the Social Determinants of Health et al., 2016). Although adherence to, and use of, malaria preventive measures depend on multiple factors such as accessibility of LLINs, the behaviour and perception of people about the risk of malaria severity and effectiveness of preventive measures play key roles. The WHO resolved to 'continue to invest in changing people's behaviour' towards ending the malaria epidemic by 2030 (World Health Organization, 2018).

To date, studies have focused on malaria prevention and the use of LLINs in Nigeria, predominantly focusing on the ownership and use of LLINs (Adebayo et al., 2014; Adedokun \& Uthman, 2020). However, there is paucity of information on the relationship between perceived severity of malaria and the use of LLINs. In some settings, uncomplicated malaria is becoming a 'normalized illness' and affects people's perception of risk (Jones \& Williams, 2004; Giardina et al., 2014). With seasonality being a source of bias, different surveys conducted during dry and hot months (e.g. because of issues of accessibility of remote areas), when people are less likely to sleep under bed nets, may affect the accuracy of the research results that inform policy decisions (Giardina et al., 2014). Also, the occurrence of malaria is closely related to naturally existing environmental and climatic conditions and anthropogenic activities (Odongo-Aginya et al., 2005). Although Nigeria's current malaria control situation has improved, with the aid of both international and local initiatives, one of the roadblocks, however, to reduction and long-term elimination is the vector harbourage posed by clogged drainage systems (Farid, 2016). Nigeria has predominantly two seasons. In the south, the rainy season lasts from March to November, whereas in the north it lasts only 
from mid-May to September (Britannica, 2021). The rest of the months are dry season. During the rainy seasons, waste accumulated in the dry seasons blocks drainage, making it hard for water to flow easily and causing stagnant water to collect, creating breeding sites for mosquitoes (Amoran et al., 2014).

Therefore, this study explored the rural-urban dimensions of the perception of malaria severity and practice of malaria preventive measures using the nationally representative 2018 Demographic and Health Survey dataset for Nigeria. The theoretical frameworks of the study were the Health Belief Model (HBM) and the Social Determinants of Health (SDH) framework, which were used to explain the results and how they inform policies towards reducing malaria incidence. The HBM is a psychological model that attempts to explain and predict health behaviours by focusing on the attitudes and beliefs of individuals (Green \& Murphy, 2014; Jones et al., 2015). It proposes six constructs to explain and predict preventive health behaviours: modifying factors, perceived threats (severity and susceptibility), benefits, barriers, self-efficacy and cues to action. It focuses on intra-personal factors, including risk-related beliefs that influence individuals' health-related decision-making. The HBM can be used to interpret people's perception of health threats and subsequent compliance with health interventions (Green \& Murphy, 2014; Watanabe et al., 2014; Jones et al., 2015).

This study explored the association of women's perceptions of the severity of malaria and the decision-making process in the uptake of preventive measures in Nigeria. The findings will inform policymakers when designing specific interventions to change the perception of the use of LLINs. The aim was to establish a baseline for effective and cost-effective initiatives for the Federal Government of Nigeria towards reducing malaria and its associated complications.

\section{Methods}

\section{Data}

The study used a female dataset from the 2018 Nigeria Demographic and Health Survey (NDHS). The 2018 NDHS data were cross-sectional and collected from 14th August to 29th December 2018. The survey adopted a two-stage sampling design (National Population Commission \& ICF, 2019), details of which can be found in previous reports (National Population Commission \& ICF, 2009; National Population Commission \& ICF, 2013). A total of 42,121 women of reproductive age (15-49 years) were eligible to be interviewed and $99.288 \%$ of them responded to the questionnaire (National Population Commission \& ICF, 2019). A total of 1128 (2.7\% of 41,821) respondents who indicated that they did not know, or were not sure, that malaria led to death were excluded from the dataset during the data cleaning before analysis. The final analytic sample was 40,693 women.

\section{Measures}

\section{Dependent variable}

The dependent variable was 'adoption of a malaria preventive measure'. The survey variable 'type of mosquito bed net(s) slept under last night' was used, with the responses ' 0 ' for no net, ' 1 ' for only a treated net, ' 2 ' for both treated and untreated nets and ' 3 ' for only an untreated net. Respondents who slept only under a treated net the night before the survey were treated as 'having adopted a malaria preventive measure' and all other respondents as otherwise.

\section{Independent variable}

The independent variable for the study was 'perceived severity of malaria'. The survey variable used was whether respondents agreed or disagreed (or responded 'do not know') to the statement 'every case of malaria can potentially lead to death' (National Population Commission \& ICF, 2019). The study hypothesized that respondents who agreed that malaria could potentially lead to death would adopt malaria preventive measures. 
Table 1. Multicollinearity statistics of the variables in the study models

\begin{tabular}{|c|c|c|c|c|c|c|}
\hline \multirow[b]{2}{*}{ Independent variable } & \multicolumn{2}{|c|}{ Full Model } & \multicolumn{2}{|c|}{ Urban Model } & \multicolumn{2}{|c|}{ Rural Model } \\
\hline & VIF & $1 / \mathrm{VIF}$ & VIF & $1 / \mathrm{VIF}$ & VIF & $1 / \mathrm{VIF}$ \\
\hline Malaria can lead to death & 1.01 & 0.990535 & 1.01 & 0.986939 & 1.01 & 0.988295 \\
\hline Has child under 5 years & 1.29 & 0.772799 & 1.26 & 0.792788 & 1.31 & 0.763548 \\
\hline Age & 1.6 & 0.626466 & 1.48 & 0.675129 & 1.81 & 0.552471 \\
\hline Education level & 2.02 & 0.495132 & 1.98 & 0.505154 & 1.5 & 0.666973 \\
\hline Marital status & 1.85 & 0.539314 & 1.69 & 0.590697 & 2.11 & 0.474416 \\
\hline Employment status & 1.1 & 0.910853 & 1.1 & 0.907664 & 1.11 & 0.900271 \\
\hline Religion & 1.31 & 0.763517 & 1.42 & 0.702679 & 1.2 & 0.834643 \\
\hline Rural-urban place of residence & 1.39 & 0.719646 & & & & \\
\hline Household wealth & 2.01 & 0.497795 & 1.6 & 0.623605 & 1.34 & 0.745936 \\
\hline Region of residence & 1.21 & 0.825977 & 1.17 & 0.852534 & 1.14 & 0.880199 \\
\hline Mean VIF & 1.48 & & 1.41 & & 1.39 & \\
\hline
\end{tabular}

\section{Control variables}

Control variables were identified and selected from the dataset based on the literature and their availability in the female dataset and included respondent's age, education level, marital status, whether they had a child under 5 years, employment status, religion, rural-urban place of residence, household wealth and region of residence.

\section{Analysis}

Analyses were conducted using STATA version 14. The variables were first cleaned and re-coded to create the final respondent sample of 40,693 women. Assumptions of multicollinearity were tested for, and no violations were observed; all independent variables in the models had variance inflation factors (VIFs) values of less than 3, which is far less than the cut-off value of 10 (see Table 1).

The svyset command was used to set the analytical environment to account for the two-stage sampling methodology (sampling weight, stratification and the primary sample units) of the NDHS. Frequency and percentages were used to describe the sample. Poisson regression was used to test the study hypothesis using bivariable and multivariable analyses. Poisson regression was used to estimate prevalence ratios because the proportion of the outcome of interest exceeded 12\% (Barros \& Hirakata, 2003; Zou, 2004; Santos et al., 2008; Zou \& Donner, 2013). In such situations, prevalence ratios should be reported instead of the frequently reported odds ratio; it has been proven that the use of odds ratios biased the coefficients showing strength and direction of statistical relationships and the confident intervals and standard errors (Barros \& Hirakata, 2003; Zou, 2004; Santos et al., 2008; Zou \& Donner, 2013).

\section{Results}

\section{Characteristics of respondents by their use of treated bed nets}

Table 2 presents the demographic and socioeconomic characteristics of the respondents by whether they slept under an LLIN the night before the survey. About $48 \%$ of respondents slept under LLINs the night before the survey. About 34\% disagreed that malaria could lead to death. 
Table 2. Distribution of characteristics of respondents by whether they reported sleeping under a long-lasting insecticidetreated net (LLIN) the night before the survey

\begin{tabular}{|c|c|c|c|}
\hline & \multirow[b]{2}{*}{$N(\%)^{\mathrm{a}}$} & \multicolumn{2}{|c|}{ Slept under LLIN } \\
\hline & & No $(\%)^{b}$ & Yes $(\%)^{b}$ \\
\hline Total & 40,693 & 52.38 & 47.62 \\
\hline Malaria can lead to death & $p \leq 0.001$ & & \\
\hline Yes & $27,023(66.4)$ & 51.11 & 48.89 \\
\hline No & $13,670(33.6)$ & 54.90 & 45.10 \\
\hline Age (years) & $p \leq 0.001$ & & \\
\hline $15-19$ & $8038(19.8)$ & 58.06 & 41.94 \\
\hline $20-24$ & $6648(16.3)$ & 48.61 & 51.39 \\
\hline $25-29$ & $7118(17.5)$ & 48.78 & 51.22 \\
\hline $30-34$ & $6055(14.9)$ & 50.62 & 49.38 \\
\hline $35-39$ & $5352(13.2)$ & 54.16 & 45.84 \\
\hline $40-44$ & $3850(9.5)$ & 54.46 & 45.54 \\
\hline $45-49$ & $3631(8.9)$ & 51.93 & 48.07 \\
\hline Education level & $p \leq 0.001$ & & \\
\hline No education & $14,274(35.1)$ & 36.92 & 63.08 \\
\hline Primary & $5883(14.5)$ & 52.33 & 47.67 \\
\hline Secondary & $15,998(39.3)$ & 62.70 & 37.30 \\
\hline Higher & $4538(11.2)$ & 64.74 & 35.26 \\
\hline Relationship status & $p \leq 0.001$ & & \\
\hline Never in union & $10,042(24.7)$ & 68.69 & 31.31 \\
\hline Currently in union/living with a man & $28,546(70.2)$ & 45.98 & 54.02 \\
\hline Formerly in union/living with a man & $2106(5.2)$ & 61.50 & 38.50 \\
\hline Has child under 5 years & $p \leq 0.001$ & & \\
\hline No & $19,219(47.2)$ & 61.79 & 38.21 \\
\hline Yes & $21,474(52.8)$ & 43.97 & 56.03 \\
\hline Employment status & $p \leq 0.001$ & & \\
\hline Not employed & $12,783(31.4)$ & 48.94 & 51.06 \\
\hline All year & $21,108(51.9)$ & 54.60 & 45.40 \\
\hline Seasonal/occasional & $6802(16.7)$ & 51.97 & 48.03 \\
\hline Religion & $p \leq 0.001$ & & \\
\hline Traditionalist/other & $231(0.6)$ & 56.12 & 43.88 \\
\hline Catholic & $4197(10.3)$ & 64.48 & 35.52 \\
\hline Other Christian & $14,367(35.3)$ & 65.12 & 34.88 \\
\hline Islam & $21,897(53.8)$ & 41.67 & 58.33 \\
\hline Place of residence & $p \leq 0.001$ & & \\
\hline Urban & $18,577(45.7)$ & 62.58 & 37.42 \\
\hline Rural & $22,116(54.4)$ & 43.82 & 56.18 \\
\hline
\end{tabular}


Table 2. (Continued)

\begin{tabular}{|c|c|c|c|}
\hline & \multirow[b]{2}{*}{$N(\%)^{\mathrm{a}}$} & \multicolumn{2}{|c|}{ Slept under LLIN } \\
\hline & & No $(\%)^{b}$ & Yes $(\%)^{b}$ \\
\hline Household wealth & $p \leq 0.001$ & & \\
\hline Poorest & $7032(17.3)$ & 36.95 & 63.05 \\
\hline Poorer & $7856(19.3)$ & 40.63 & 59.37 \\
\hline Middle & $8028(19.7)$ & 48.76 & 51.24 \\
\hline Richer & $8730(21.5)$ & 61.30 & 38.70 \\
\hline Richest & $9047(22.2)$ & 69.20 & 30.80 \\
\hline Region of residence & $p \leq 0.001$ & & \\
\hline North Central & $5825(14.3)$ & 56.60 & 43.40 \\
\hline North East & $6479(15.9)$ & 50.08 & 49.92 \\
\hline North West & $12,008(29.5)$ & 26.71 & 73.29 \\
\hline South East & $4762(11.7)$ & 68.31 & 31.69 \\
\hline South South & 4762 (11.6) & 71.11 & 28.89 \\
\hline South West & $6914(17.0)$ & 71.87 & 28.13 \\
\hline
\end{tabular}

acolumn percentage reported.

${ }^{\mathrm{b}}$ Row percentage reported.

About $49 \%$ of the women who agreed that malaria could lead to death slept under LLINs, compared with $45 \%$ of those who disagreed. Women in the 20-24 (51.39\%) and 25-29 (51.22\%) age categories had higher proportions of those sleeping under LLINs the previous night than women in other age groups. Surprisingly, more women with no formal education (63.08\%) slept under LLINs the previous night compared with those with some formal education (less than 50\%). Women living in rural areas were more likely to have slept under LLINs the previous night $(56.18 \%)$ than their urban counterparts $(37.42 \%)$. All the analysed socio-demographic variables were significantly associated with the outcome. Details of the remaining statistics are shown in Table 2.

\section{Regression analysis of perceived malaria severity by use of treated bed nets}

Women who perceived that malaria could lead to death had a higher likelihood of sleeping under LLINs the previous night in the urban, rural and combined samples (Table 3). After adjusting for other socio-demographic variables, the association between perceived malaria severity and use of LLINs was only significant for rural women (APR $=0.964,95 \%$ CI: $0.933,0.996$ ) (Table 3). The results unexpectedly suggest that, holding the effect of all other variables in the model constant, rural Nigerian women who perceived malaria to be severe (and could lead to death) had a lower likelihood of adopting a malaria-preventive measure compared with those who did not perceive malaria to be severe (Table 3 ).

Rural-urban variations were observed in the relationship between socio-demographic variables and the use of LLINs. Women's age was significantly associated with sleeping under an LLIN in urban areas but not in rural areas. In the urban sample, compared with 15- to 19-year-old women, women who belonged to an older age group (except 20-24 years and 45-49 years) were significantly less likely to sleep under an LLIN (Table 3). Although formal education was a significant factor in both urban and rural models, some differences existed in their contribution to the model. In the rural model, women who attained a secondary level education or higher were more likely to use an LLIN. In the urban model, however, only the attainment of a post-secondary education 
Table 3. Perceived malaria severity regressed on the use of long-lasting insecticide-treated nets and controlled for socio-demographic variables

\begin{tabular}{|c|c|c|c|c|c|c|}
\hline & \multicolumn{2}{|c|}{ Full sample } & \multicolumn{2}{|c|}{ Urban } & \multicolumn{2}{|c|}{ Rural } \\
\hline & $\mathrm{PR}[95 \% \mathrm{Cl}]$ & APR $[95 \% \mathrm{Cl}]$ & PR $[95 \% \mathrm{Cl}]$ & APR $[95 \% \mathrm{Cl}]$ & $\mathrm{PR}[95 \% \mathrm{Cl}]$ & APR $[95 \% \mathrm{Cl}]$ \\
\hline \multicolumn{7}{|c|}{ Malaria can lead to death } \\
\hline \multicolumn{7}{|l|}{ No (Ref.) } \\
\hline Yes & $\begin{array}{c}1.084^{\star \star \star} \\
{[1.045,1.125]}\end{array}$ & $\begin{array}{c}0.992 \\
{[0.963,1.023]}\end{array}$ & $\begin{array}{c}1.197^{\star \star \star} \\
{[1.118,1.282]}\end{array}$ & $\begin{array}{c}1.046 \\
{[0.981,1.115]}\end{array}$ & $\begin{array}{c}1.050^{\star} \\
{[1.009,1.092]}\end{array}$ & $\begin{array}{c}0.964^{*} \\
{[0.933,0.996]}\end{array}$ \\
\hline \multicolumn{7}{|l|}{ Age (years) } \\
\hline \multicolumn{7}{|l|}{ 15-19 (Ref.) } \\
\hline $20-24$ & $\begin{array}{c}1.225^{\star \star \star} \\
{[1.173,1.280]}\end{array}$ & $\begin{array}{c}0.988 \\
{[0.944,1.034]}\end{array}$ & $\begin{array}{c}1.196^{\star \star \star} \\
{[1.090,1.311]}\end{array}$ & $\begin{array}{c}0.933 \\
{[0.842,1.033]}\end{array}$ & $\begin{array}{c}1.225^{\star \star \star} \\
{[1.170,1.282]}\end{array}$ & $\begin{array}{c}1.025 \\
{[0.980,1.073}\end{array}$ \\
\hline $25-29$ & $\begin{array}{c}1.221^{\star \star \star} \\
{[1.167,1.277]}\end{array}$ & $\begin{array}{c}0.964 \\
{[0.920,1.009]}\end{array}$ & $\begin{array}{c}1.225^{\star \star \star} \\
{[1.115,1.345]}\end{array}$ & $\begin{array}{c}0.880^{*} \\
{[0.787,0.984]}\end{array}$ & $\begin{array}{c}1.221^{\star \star \star} \\
{[1.165,1.279]}\end{array}$ & $\begin{array}{c}1.013 \\
{[0.970,1.057]}\end{array}$ \\
\hline $30-34$ & $\begin{array}{c}1.177^{\star \star \star} \\
{[1.124,1.234]}\end{array}$ & $\begin{array}{c}0.940^{*} \\
{[0.895,0.987]}\end{array}$ & $\begin{array}{c}1.255^{\star \star \star} \\
{[1.146,1.375]}\end{array}$ & $\begin{array}{c}0.885^{\star} \\
{[0.789,0.994]}\end{array}$ & $\begin{array}{c}1.159^{\star \star \star} \\
{[1.103,1.218]}\end{array}$ & $\begin{array}{c}0.970 \\
{[0.924,1.019}\end{array}$ \\
\hline $35-39$ & $\begin{array}{c}1.093^{\star \star \star} \\
{[1.042,1.147]}\end{array}$ & $\begin{array}{c}0.906^{\star \star *} \\
{[0.863,0.952]}\end{array}$ & $\begin{array}{c}1.107^{\star} \\
{[1.017,1.205]}\end{array}$ & $\begin{array}{c}0.811^{\star \star \star} \\
{[0.727,0.904]}\end{array}$ & $\begin{array}{c}1.114^{\star \star \star} \\
{[1.054,1.178]}\end{array}$ & $\begin{array}{c}0.968 \\
{[0.918,1.021]}\end{array}$ \\
\hline $40-44$ & $\begin{array}{c}1.086^{\star \star} \\
{[1.030,1.145]}\end{array}$ & $\begin{array}{c}0.898^{\star \star *} \\
{[0.851,0.948]}\end{array}$ & $\begin{array}{c}1.063 \\
{[0.956,1.182]}\end{array}$ & $\begin{array}{c}0.795^{\star \star \star} \\
{[0.695,0.910]}\end{array}$ & $\begin{array}{c}1.100^{\star \star \star} \\
{[1.043,1.161]}\end{array}$ & $\begin{array}{c}0.959 \\
{[0.912,1.009}\end{array}$ \\
\hline $45-49$ & $\begin{array}{c}1.146^{\star \star \star} \\
{[1.090,1.205]}\end{array}$ & $\begin{array}{c}0.993 \\
{[0.940,1.049]}\end{array}$ & $\begin{array}{c}1.211^{\star \star \star} \\
{[1.103,1.330]}\end{array}$ & $\begin{array}{c}0.943 \\
{[0.829,1.071]}\end{array}$ & $\begin{array}{c}1.120^{\star \star \star} \\
{[1.060,1.183]}\end{array}$ & $\begin{array}{c}1.014 \\
{[0.957,1.074]}\end{array}$ \\
\hline \multicolumn{7}{|c|}{ Education level } \\
\hline \multicolumn{7}{|c|}{ No education (Ref.) } \\
\hline Primary & $\begin{array}{c}0.756^{\star \star \star} \\
{[0.723,0.790]}\end{array}$ & $\begin{array}{c}1.052^{\star} \\
{[1.009,1.096]}\end{array}$ & $\begin{array}{c}0.744^{* * *} \\
{[0.680,0.813]}\end{array}$ & $\begin{array}{c}1.059 \\
{[0.974,1.152]}\end{array}$ & $\begin{array}{c}0.815^{\star * \star} \\
{[0.777,0.855]}\end{array}$ & $\begin{array}{c}1.045 \\
{[0.998,1.095]}\end{array}$ \\
\hline Secondary & $\begin{array}{c}0.591^{\star \star \star} \\
{[0.565,0.619]}\end{array}$ & $\begin{array}{c}1.066^{\star \star} \\
{[1.020,1.115]}\end{array}$ & $\begin{array}{c}0.621^{\star \star \star} \\
{[0.568,0.678]}\end{array}$ & $\begin{array}{c}1.067 \\
{[0.983,1.158]}\end{array}$ & $\begin{array}{c}0.665^{\star \star \star} \\
{[0.629,0.703]}\end{array}$ & $\begin{array}{c}1.072^{\star *} \\
{[1.019,1.127]}\end{array}$ \\
\hline Higher & $\begin{array}{c}0.559^{\star \star \star} \\
{[0.517,0.604]}\end{array}$ & $\begin{array}{c}1.153^{\star \star \star} \\
{[1.074,1.237]}\end{array}$ & $\begin{array}{c}0.638^{\star \star \star} \\
{[0.565,0.720]}\end{array}$ & $\begin{array}{c}1.159^{\star \star} \\
{[1.047,1.284]}\end{array}$ & $\begin{array}{c}0.598^{\star \star \star} \\
{[0.536,0.668]}\end{array}$ & $\begin{array}{c}1.144^{\star \star} \\
{[1.047,1.250]}\end{array}$ \\
\hline
\end{tabular}


Table 3. (Continued)

\begin{tabular}{|c|c|c|c|c|c|c|}
\hline & \multicolumn{2}{|c|}{ Full sample } & \multicolumn{2}{|c|}{ Urban } & \multicolumn{2}{|c|}{ Rural } \\
\hline & $\mathrm{PR}[95 \% \mathrm{Cl}]$ & APR $[95 \% \mathrm{Cl}]$ & $\mathrm{PR}[95 \% \mathrm{Cl}]$ & APR $[95 \% \mathrm{Cl}]$ & $\mathrm{PR}[95 \% \mathrm{Cl}]$ & $\operatorname{APR}[95 \% \mathrm{Cl}]$ \\
\hline \multicolumn{7}{|l|}{ Relationship status } \\
\hline \multicolumn{7}{|l|}{ Never in union (Ref.) } \\
\hline Currently in union/living with a man & $\begin{array}{c}1.725^{\star \star \star} \\
{[1.648,1.806]}\end{array}$ & $\begin{array}{c}1.423^{\star \star \star} \\
{[1.348,1.502]}\end{array}$ & $\begin{array}{c}1.572^{\star \star \star} \\
{[1.461,1.690]}\end{array}$ & $\begin{array}{c}1.479^{\star \star \star} \\
{[1.324,1.653]}\end{array}$ & $\begin{array}{c}1.668^{\star \star \star} \\
{[1.580,1.761]}\end{array}$ & $\begin{array}{c}1.390^{\star \star \star} \\
{[1.307,1.478]}\end{array}$ \\
\hline Formerly in union/living with a man & $\begin{array}{c}1.229^{\star \star \star} \\
{[1.139,1.327]}\end{array}$ & $\begin{array}{c}1.223^{\star \star \star} \\
{[1.131,1.322]}\end{array}$ & $\begin{array}{c}1.236^{\star \star \star} \\
{[1.102,1.387]}\end{array}$ & $\begin{array}{c}1.239^{\star *} \\
{[1.082,1.419]}\end{array}$ & $\begin{array}{c}1.198^{\star \star \star} \\
{[1.089,1.318]}\end{array}$ & $\begin{array}{c}1.232^{\star \star \star} \\
{[1.114,1.363]}\end{array}$ \\
\hline \multicolumn{7}{|l|}{ Has child under 5 years } \\
\hline \multicolumn{7}{|l|}{ No (Ref.) } \\
\hline Yes & $\begin{array}{c}1.466^{\star \star \star} \\
{[1.417,1.518]}\end{array}$ & $\begin{array}{c}1.123^{\star \star \star} \\
{[1.085,1.163]}\end{array}$ & $\begin{array}{c}1.499^{\star \star \star} \\
{[1.409,1.596]}\end{array}$ & $\begin{array}{c}1.214^{\star \star \star} \\
{[1.126,1.308]}\end{array}$ & $\begin{array}{c}1.347^{\star \star \star} \\
{[1.300,1.395]}\end{array}$ & $\begin{array}{c}1.069^{\star \star \star} \\
{[1.033,1.106]}\end{array}$ \\
\hline \multicolumn{7}{|l|}{ Employment status } \\
\hline \multicolumn{7}{|l|}{ Not employed (Ref.) } \\
\hline All year & $\begin{array}{c}0.889^{\star \star \star} \\
{[0.859,0.920]}\end{array}$ & $\begin{array}{c}1.014 \\
{[0.984,1.045]}\end{array}$ & $\begin{array}{c}0.918^{\star \star} \\
{[0.864,0.975]}\end{array}$ & $\begin{array}{c}0.996 \\
{[0.940,1.056]}\end{array}$ & $\begin{array}{c}0.927^{\star \star \star} \\
{[0.894,0.962]}\end{array}$ & $\begin{array}{c}1.018 \\
{[0.984,1.053]}\end{array}$ \\
\hline Seasonal/occasional & $\begin{array}{c}0.941^{\star} \\
{[0.896,0.988]}\end{array}$ & $\begin{array}{c}0.978 \\
{[0.937,1.021]}\end{array}$ & $\begin{array}{c}1.040 \\
{[0.955,1.133]}\end{array}$ & $\begin{array}{c}1.090^{\star} \\
{[1.005,1.182]}\end{array}$ & $\begin{array}{c}0.849^{\star \star \star} \\
{[0.802,0.899]}\end{array}$ & $\begin{array}{c}0.943^{*} \\
{[0.898,0.991]}\end{array}$ \\
\hline \multicolumn{7}{|l|}{ Religion } \\
\hline \multicolumn{7}{|l|}{ Traditionalist/other (Ref.) } \\
\hline Catholic & $\begin{array}{c}0.809 \\
{[0.553,1.185]}\end{array}$ & $\begin{array}{c}1.018 \\
{[0.842,1.231]}\end{array}$ & $\begin{array}{c}1.001 \\
{[0.637,1.572]}\end{array}$ & $\begin{array}{c}1.001 \\
{[0.652,1.536]}\end{array}$ & $\begin{array}{c}0.864 \\
{[0.577,1.294]}\end{array}$ & $\begin{array}{c}1.061 \\
{[0.856,1.315]}\end{array}$ \\
\hline Other Christian & $\begin{array}{c}0.795 \\
{[0.545,1.158]}\end{array}$ & $\begin{array}{c}1.042 \\
{[0.869,1.251]}\end{array}$ & $\begin{array}{c}1.055 \\
{[0.671,1.659]}\end{array}$ & $\begin{array}{c}1.170 \\
{[0.758,1.805]}\end{array}$ & $\begin{array}{c}0.783 \\
{[0.526,1.166]}\end{array}$ & $\begin{array}{c}0.988 \\
{[0.810,1.205]}\end{array}$ \\
\hline Islam & $\begin{array}{c}1.329 \\
{[0.910,1.941]}\end{array}$ & $\begin{array}{c}1.022 \\
{[0.852,1.227]}\end{array}$ & $\begin{array}{c}1.580^{\star} \\
{[1.011,2.469]}\end{array}$ & $\begin{array}{c}1.112 \\
{[0.717,1.726]}\end{array}$ & $\begin{array}{c}1.308 \\
{[0.877,1.950]}\end{array}$ & $\begin{array}{c}1.010 \\
{[0.827,1.233]}\end{array}$ \\
\hline
\end{tabular}


Table 3. (Continued)

\begin{tabular}{|c|c|c|c|c|c|c|}
\hline & \multicolumn{2}{|c|}{ Full sample } & \multicolumn{2}{|c|}{ Urban } & \multicolumn{2}{|c|}{ Rural } \\
\hline & $\mathrm{PR}[95 \% \mathrm{Cl}]$ & APR $[95 \% \mathrm{Cl}]$ & $\mathrm{PR}[95 \% \mathrm{Cl}]$ & APR $[95 \% \mathrm{Cl}]$ & $\mathrm{PR}[95 \% \mathrm{Cl}]$ & APR $[95 \% \mathrm{Cl}]$ \\
\hline \multicolumn{7}{|l|}{ Place of residence } \\
\hline Urban (Ref.) & & & - & & - & \\
\hline Rural & $\begin{array}{c}1.501^{\star \star \star} \\
{[1.421,1.586]}\end{array}$ & $\begin{array}{c}1.100^{\star \star \star} \\
{[1.044,1.159]}\end{array}$ & - & & - & \\
\hline \multicolumn{7}{|l|}{ Household wealth } \\
\hline \multicolumn{7}{|l|}{ Poorest (Ref.) } \\
\hline Poorer & $\begin{array}{c}0.942^{*} \\
{[0.894,0.992]}\end{array}$ & $\begin{array}{c}0.986 \\
{[0.938,1.036]}\end{array}$ & $\begin{array}{c}0.983 \\
{[0.857,1.127]}\end{array}$ & $\begin{array}{c}0.965 \\
{[0.848,1.098]}\end{array}$ & $\begin{array}{c}0.947 \\
{[0.895,1.003]}\end{array}$ & $\begin{array}{c}0.977 \\
{[0.926,1.031]}\end{array}$ \\
\hline Middle & $\begin{array}{c}0.813^{* * *} \\
{[0.765,0.863]}\end{array}$ & $\begin{array}{c}1.009 \\
{[0.955,1.066]}\end{array}$ & $\begin{array}{c}0.824^{\star} \\
{[0.697,0.974]}\end{array}$ & $\begin{array}{c}0.878 \\
{[0.766,1.006]}\end{array}$ & $0.868^{\star \star \star}[0.812,0.928]$ & $\begin{array}{c}1.035 \\
{[0.976,1.099]}\end{array}$ \\
\hline Richer & $\begin{array}{c}0.614^{\star \star \star} \\
{[0.570,0.661]}\end{array}$ & $\begin{array}{c}0.890^{\star \star \star} \\
{[0.831,0.954]}\end{array}$ & $\begin{array}{c}0.663^{\star * *} \\
{[0.555,0.791]}\end{array}$ & $\begin{array}{c}0.793^{\star *} \\
{[0.689,0.914]}\end{array}$ & $\begin{array}{c}0.687^{\star \star \star} \\
{[0.630,0.748]}\end{array}$ & $\begin{array}{c}0.946 \\
{[0.873,1.025]}\end{array}$ \\
\hline Richest & $\begin{array}{c}0.489^{\star * *} \\
{[0.448,0.532]}\end{array}$ & $0.776^{\star \star \star}[0.710,0.849]$ & $\begin{array}{c}0.564^{\star \star \star} \\
{[0.472,0.675]}\end{array}$ & $\begin{array}{c}0.731^{\star \star \star} \\
{[0.626,0.855]}\end{array}$ & $\begin{array}{c}0.483^{\star \star *} \\
{[0.418,0.559]}\end{array}$ & $\begin{array}{c}0.773^{\star \star \star} \\
{[0.677,0.884]}\end{array}$ \\
\hline \multicolumn{7}{|c|}{ Region of residence } \\
\hline North Central & $\begin{array}{c}1.543^{\star \star \star} \\
{[1.390,1.713]}\end{array}$ & $1.358^{\star \star \star}[1.226,1.505]$ & $\begin{array}{c}1.503^{\star \star \star} \\
{[1.288,1.753]}\end{array}$ & $\begin{array}{c}1.452^{\star \star \star} \\
{[1.244,1.696]}\end{array}$ & $\begin{array}{c}1.091 \\
{[0.959,1.242]}\end{array}$ & $\begin{array}{c}1.062 \\
{[0.941,1.198}\end{array}$ \\
\hline North East & $\begin{array}{c}1.775^{\star \star \star} \\
{[1.595,1.975]}\end{array}$ & $\begin{array}{c}1.517^{\star \star \star} \\
{[1.365,1.685]}\end{array}$ & $\begin{array}{c}1.789^{\star \star \star} \\
{[1.529,2.092]}\end{array}$ & $\begin{array}{c}1.711^{\star \star \star} \\
{[1.467,1.995]}\end{array}$ & $\begin{array}{c}1.223^{\star \star} \\
{[1.073,1.395]}\end{array}$ & $\begin{array}{c}1.186^{\star *} \\
{[1.047,1.343]}\end{array}$ \\
\hline North West & $\begin{array}{c}2.606^{\star \star *} \\
{[2.375,2.859]}\end{array}$ & $\begin{array}{c}2.199^{\star \star \star} \\
{[1.998,2.421]}\end{array}$ & $\begin{array}{c}2.574^{\star \star \star} \\
{[2.281,2.905]}\end{array}$ & $\begin{array}{c}2.393^{\star \star \star} \\
{[2.107,2.717]}\end{array}$ & $\begin{array}{c}1.817^{\star \star \star} \\
{[1.617,2.042]}\end{array}$ & $\begin{array}{c}1.723^{\star \star \star} \\
{[1.538,1.930]}\end{array}$ \\
\hline South East & $\begin{array}{c}1.127 \\
{[0.991,1.281]}\end{array}$ & $\begin{array}{c}1.094 \\
{[0.957,1.252]}\end{array}$ & $\begin{array}{c}1.322^{\star \star *} \\
{[1.129,1.548]}\end{array}$ & $\begin{array}{c}1.306^{\star \star} \\
{[1.092,1.562]}\end{array}$ & $\begin{array}{c}0.687^{\star \star \star} \\
{[0.595,0.795]}\end{array}$ & $\begin{array}{c}0.707^{\star \star \star} \\
{[0.614,0.815]}\end{array}$ \\
\hline South South & $\begin{array}{c}1.027 \\
{[0.905,1.165]}\end{array}$ & $\begin{array}{c}0.978 \\
{[0.867,1.103]}\end{array}$ & $\begin{array}{c}1.117 \\
{[0.934,1.337]}\end{array}$ & $\begin{array}{c}1.128 \\
{[0.946,1.346]}\end{array}$ & $\begin{array}{c}0.699^{\star \star \star} \\
{[0.595,0.821]}\end{array}$ & $\begin{array}{c}0.731^{\star \star \star} \\
{[0.628,0.851]}\end{array}$ \\
\hline
\end{tabular}


Table 3. (Continued)

\begin{tabular}{|c|c|c|c|c|c|c|}
\hline & \multicolumn{2}{|c|}{ Full sample } & \multicolumn{2}{|c|}{ Urban } & \multicolumn{2}{|c|}{ Rural } \\
\hline & PR $[95 \% \mathrm{Cl}]$ & APR $[95 \% \mathrm{Cl}]$ & PR $[95 \% \mathrm{Cl}]$ & APR $[95 \% \mathrm{Cl}]$ & PR $[95 \% \mathrm{Cl}]$ & APR $[95 \% \mathrm{Cl}]$ \\
\hline \multicolumn{7}{|l|}{ Model details } \\
\hline No. strata & & 74 & & 37 & & 37 \\
\hline No. observations & & 40,693 & & 16,506 & & 24,187 \\
\hline Population size & & $40,634.438$ & & 18,550 & & 22,084 \\
\hline No. PSUs & & 1389 & & 576 & & 813 \\
\hline \multirow[t]{2}{*}{ Design df } & & 1315 & & 539 & & 776 \\
\hline & & $F(28,1288)=58.35$ & & $F(27,513)=28.04$ & & $F(27,750)=39.17$ \\
\hline Prob $>F$ & & 0.0000 & & 0.0000 & & 0.0000 \\
\hline
\end{tabular}

Exponentiated coefficients; 95\% confidence intervals in brackets.

${ }^{\star} p<0.05 ;{ }^{\star \star} p<0.01 ;{ }^{\star \star \star} p<0.001$ 
status was positively associated with sleeping under an LLIN (Table 3). Also, marital status and having an under- 5 child were positively associated with sleeping under an LLIN in both urban and rural models, but with slightly different effects (Table 3 ). The effect of being currently married or formerly married or having an under- 5 child was slightly higher among urban women than rural women. Compared with unemployed women, women who were seasonally or occasionally employed were significantly more likely to sleep under an LLIN in urban areas but less likely to sleep under an LLIN in rural areas (Table 3). Household wealth was negatively associated with sleeping under LLINs in both urban and rural areas, but with some differences. Belonging to the richer or richest households was negatively associated with using an LLIN compared with the poorest households (Table 3). In rural areas, however, belonging to only the richest households was negatively associated with the use of LLINs. Region of residence was also significantly associated with the use of LLINs, but with rural-urban variation. Compared with the South West region of Nigeria, the prevalence of women's use of LLINs was significantly higher in urban than rural areas of North Central, North East, North West and South East (Table 3). For urban women, residing in the South South region was not significantly associated with the use of LLINs. For rural women, residing in the South East or South South region was significantly associated with a lower likelihood of using the LLINs. Religion was not a significant contributor to either the urban or rural models (Table 3).

\section{Discussion}

By applying the Social Determinants of Health (SDH) framework and Health Belief Model (HBM), this study sought to examine the rural-urban dimensions of the perception of malaria severity and practice of malaria preventive measures using the 2018 Nigeria Demographic and Health Survey dataset. Almost half (49\%) of the surveyed women who had the perception that malaria could lead to death slept under long-lasting insecticide-treated nets (LLINs) the night before the survey. Multiple reasons for non-use of LLINs, including discomfort due to heat and difficulty in hanging up the nets, have been reported elsewhere (Adebayo et al., 2014). Women's likelihood of sleeping under an LLIN the night before the survey was significantly associated with their age, education level, marital status, having a child under 5 years, employment status, religion, place of residence (urban/rural), household wealth and region of residence. Although the perception of severe malaria leading to death was significantly higher, compared with the perception of severe malaria not leading to death, across both urban and rural residence, adjusting for the above socio-demographic factors resulted in significant rural-urban differences.

The study found that Nigerian women's perception of the severity of malaria influences their use of LLINs differently in urban compared with rural areas. Specifically, having the perception that malaria could lead to death had no association with the use of LLINs in urban areas. In rural areas, however, agreeing that malaria could lead to death had an association with a lower likelihood of using LLINs. A qualitative exploration is therefore needed to understand why severity did not seem to increase the likelihood of treated bed net use in rural setting. Certain haemoglobinopathies such as sickle cell anaemia variously reduce the risk of developing malaria syndromes (Taylor et al., 2012). These haemoglobinopathies, which are also common among Nigerians, are highly prevalent in some human populations that are/were currently or historically exposed to the malaria parasite P. falciparum. For example, with a sickle haemoglobin (HbS) carrier prevalence of $25 \%$ to $40 \%$, Nigeria bears the greatest burden of sickle cell disorder worldwide (Adewoyin et al., 2019). Therefore, these haemoglobinopathies may affect the perceived severity of malaria and use of preventive measures in Nigeria. From the perspective of the HBM alone, perceived severity of malaria is positively associated with the use of LLINs. However, after accounting for other sociodemographic factors in line with the SDH framework, the positive relationship observed in the bivariable model changed into negative, indicating that perceived severity has a negative 
relationship with the use of LLINs. Due to their unavailability in the dataset, it was not possible to control for variables that mimic the other components of the HBM (perceived susceptibility, perceived benefits, cues to action and self-efficacy). Therefore, it was impossible to conclude that the HBM alone was sufficient to understand the observed phenomenon. Generally, the observed rural-urban dichotomy in the influence of perceived severity of malaria on the use of the LLINs for sleep underscores the need to account for contextual implications in designing policies that influence people's health beliefs and behaviours. Furthermore, as intimated in the SDH framework, people's health practices and health literacies are influenced by where they live and work (World Health Organization, 2010; Committee on Educating Health Professionals to Address the Social Determinants of Health et al., 2016). Therefore, this rural-urban difference would have been lost if the study did not account for it, which has been a frequent practice in similar public health publications, in addition to their lack of control for the severity of malaria (Ameyaw et al., 2020). Thus, the results also have lessons for data analysts using nationally representative data. They suggest that observation of a significant association, or lack of it, in studies that fail to account for the rural-urban divide may present an incomplete picture for policymakers.

Women's age was found to be significantly associated with the use of LLINs, but with a ruralurban effect. Whereas age was a significant factor in the association in urban areas, it was not significant in rural areas. In urban areas, however, women who were older than the 15-24 years group had a lower likelihood of using LLINs. This suggests that adolescent and younger adult women in urban areas were more likely to use LLINs. This finding supports those of similar previous studies on the age-LLIN use association (Ameyaw et al., 2020) but contradicts the findings of others (Singh et al., 2013). Most young women (15-24 years) in urban areas may be in higher education institutions and may require LLINs to protect themselves from mosquito bites in their rented accommodation (Kudom \& Mensah, 2010). Nevertheless, it is important to note that malaria can have a devastating impact on the health of women as they age. Compared with their older counterparts, younger women are more likely to use LLINs, and this may affect the drive for malaria prevention and elimination in Nigeria. Younger women constitute a large part of the population, are caregivers of under-five children, and in early child-bearing age (Babalola et al., 2019). Malaria preventive measures, in line with universal and continuous mass LLIN distribution campaigns targeting the entire population, should be intensified.

Despite formal education being significant in both urban and rural models, there were some differences in their contributions to the model. In the rural model, women who attained a secondary level education or higher were more likely to use LLINs, while this association was only significant in women with post-secondary education status in the urban setting. Although the rural-urban differences in education may not be explicitly explained, it is well-known that the opportunity for higher education is greater in urban than in rural areas, so post-secondary education may serve as a baseline qualification for most people. The role of education in influencing health behaviours is well-documented (Hahn \& Truman, 2015; Shimaponda-Mataa et al., 2017; Oladimeji et al., 2019), as is its role in increasing the likelihood that households purchase or own LLINs (Deressa \& Ali, 2009), since education and information are important drivers for change (Ezire et al., 2015). Previous studies have indicated a positive association between education on the life history of mosquitoes and on the mechanism of control strategies with the use of LLINs (Kudom \& Mensah, 2010). In contrast, other studies have reported a negative association (Adebayo et al., 2014). Therefore, policymakers need to invest significantly in educating the general population at least to the secondary level. Tuition-free education such as the Free Secondary Education policy implemented in the neighbouring country of Ghana could be adopted and/or adapted, and it needs the commitment of policymakers since such policy requires more resources.

The study found that women who were currently married or formerly married or who had under-5 children were more likely to sleep under net among urban women than rural women. Generally, being married or having a partner or having under- 5 children seems to be associated 
with positive health behaviours such as the use of LLINs at night. Previous studies have found a protective association between marital status and health-seeking behaviours (Umberson, 1992; Robards et al., 2012; Hilz \& Wagner, 2018). Conversely, unmarried women may lack spousal support and be disadvantaged in access to resources compared with their married counterparts (Nkoka et al., 2019). Under-5 children, especially those being breastfed, have a higher likelihood of sharing a bed with their parents, thereby increasing the chances of both the woman and the child sleeping under an LLIN (Auta, 2012). Women who are currently or formerly married are more likely to be pregnant and to have an under- 5 child compared with those who had never been in a union. Specifically, urban women have more access to health care facilities and antenatal services providing peri-natal services, including education on LLIN use. The availability of LLIN distribution and/or sales points, and other factors such as marital status, may favour urban women. Policymakers may need to consider implementing couple-friendly interventions to improve LLIN uptake among rural women, as well as never-in-union women.

Compared with unemployed women, women who were seasonally or occasionally employed were significantly more likely to sleep under LLINs in urban areas but less likely to sleep under LLINs in rural areas. Generally, women who are seasonally or occasionally employed are more likely to have the economic power to purchase or own an LLIN than unemployed women. However, it is difficult to explain the rural-urban differences existing among seasonally or occasionally employed women. This calls for further studies, particularly qualitative studies, to explain the differences. More importantly, government and non-governmental organizations in Nigeria must adopt policies and programmes that encourage integrating malaria preventive measures into existing work benefits and structures.

The study found that household wealth was negatively associated with sleeping under an LLIN in both urban and rural areas, but with some differences. Belonging to the richer or richest households among urban women was negatively associated with using an LLIN. In rural areas, however, belonging to only the richest households was negatively associated with the use of the LLINs. In other words, richer people were less likely to use LLINs. Generally, rich households can provide decent houses that are well-fitted with mosquito nets for doors and windows and application of mosquito repellent formulae or insecticides to prevent mosquito bites, thereby reducing their need to use LLINs. Also, women from wealthy households usually reside in environments that hinder mosquito breeding, unlike their poor counterparts (Adedokun \& Uthman, 2020). Therefore, urban women who are from richer or richest households may benefit from the protective association between household wealth and well-fitted, mosquito bite-preventive accommodation. However, regardless of the direction of the association, there is a need for the government and/or policymakers to increase efforts towards LLIN distribution mechanisms that accommodate socioeconomic differences in LLIN uptakes and use. The Nigerian government should implement pro-poor policy interventions and/or decent accommodation systems to improve rural-urban equity in ownership of decent housing, with the associated benefit of well-fitted door and window nets, as part of malaria preventive measures.

The study found region of residence to be significantly associated with the use of LLINs, but with a rural-urban difference. Previous studies in Nigeria have indicated that region of residence is the most significant predictor of LLIN use due to the heterogenous transmission of malaria across the country (Andrada et al., 2019). Different studies have reported ownership of LLINs to be either low (Eteng et al., 2014; Alawode et al., 2019) or high (Tobin-West \& Alex-Hart, 2011) in urban areas in Nigeria, and this could explain why this study found women in urban regions to have either high or low prevalences of LLIN use. Compared with the South West region, the North Central, North East, North West and South East regions had significantly higher prevalence estimates of women's LLIN use in urban than rural areas. Previous studies have indicated that northern Nigeria generally has a significantly higher incidence of malaria, and number of new malaria cases, than southern Nigeria, possibly due to poor health care access and public health services in some areas of the north and the impact of the Rivers Niger and Benue, and even urban 
agricultural development that involves irrigation (Okunlola \& Oyeyemi, 2019). Therefore, people in northern Nigeria are more likely to use LLINs than their southern counterparts. Indeed, Okunlola and Oyeyemi (2019) reported greater coverage of LLINs in the North East region. Although it appeared to be the rainy season in the south and the dry season in the north of the country during the data collection, this study's findings suggest that the seasonality of data collection may have little or no impact on LLIN use. It is expected that, during the rainy period, there will be increased breeding of mosquitoes, high exposure to malaria and a resultant, expected increase in the use of LLINs. However, these findings do not suggest an increased use of LLINs in the south, thereby suggesting that other factors may be at play beyond the season of data collection.

Regarding the higher use of LLINs among urban than rural women, poor health service delivery, lack of access and lack of health professionals posted to rural areas widen the gap for LLIN use sensitization and a resultant low use of LLINs in rural areas (Okunlola \& Oyeyemi, 2019). This study observed no significant association between religion and rural-urban residence. Therefore, there is no need for policymakers to devise interventions based on religion. However, further studies may consider regional analyses of this association to identify if there is any subnational masking.

The main strengths of this study were that is was based on a large, nationally representative survey (2018 NDHS) and used standardized, analytic methodology. This means that the findings can be generalized to the entire population. Also, it employed complex sample analyses in accounting for sampling units and weighting. In addition, it unmasked rural-urban variations in the perception of malaria severity and the practice of malaria preventive measures in Nigeria. The main limitations of this study were its use of secondary data and its cross-sectional design. Therefore, causal relationships could not be inferred between the predictors and the outcome variables. It was also restricted to variables available in the NDHS data. Also, the NDHS data were collected in a predominantly rainy period in the south and dry period in the north of Nigeria, and these differences in seasonality could possibly have affected the results.

In conclusion, the study found that about $48 \%$ of Nigerian women slept under long-lasting insecticide-treated mosquito nets the night before the 2018 survey. Women who perceived that malaria could lead to death had a higher likelihood of using a treated bed net in the urban, rural and combined samples. However, after adjusting for other socio-demographic variables, the association between respondents' perceived malaria severity and their use of treated bed nets was significant for rural Nigerian women only. Rural Nigerian women who perceived malaria to be severe had a lower likelihood of using treated bed nets. The study observed rural-urban variations in the relationship between socioeconomic and demographic variables and the use of treated bed nets. The results have implications for health policymaking towards improving the use of insecticide-treated bed nets as a malaria preventive measure, taking into consideration the socioeconomic stratifications of the Nigerian population as explained by the SDH framework, and by modifying factors, perceived threat (severity and susceptibility), benefits, barriers, self-efficacy and cues to action as postulated by the HBM. The Federal Government of Nigeria needs to implement pro-poor policies to improve wealth and educational opportunities across all regions, rural and urban areas as well as devise couple-friendly and employment-oriented strategies for malaria prevention.

Acknowledgment. The authors would like to thank the thank the Demographic and Health Survey (DHS) programme for their support and for permitting free access to the original data used in this study.

Funding. This research received no specific grant from any funding agency, commercial entity or not-for-profit organization.

Conflict of Interests. The authors have no conflicts of interest to declare.

Ethical approval. The authors obtained permission from the DHS program to use the dataset after a simple request-access registration at sprogram.com/data/dataset_admin/index.cfm. The DHS indicated that it obtained written and verbal consent from all eligible participants before their data were collected (National Population Commission \& ICF, 2019). 


\section{References}

Adebayo AM, Akinyemi OO and Cadmus EO (2014) Ownership and utilisation of insecticide-treated mosquito nets among caregivers of under-five children and pregnant women in a rural community in Southwest Nigeria. Journal of Preventive Medicine and Hygiene 55(2), 58-64.

Adedokun ST and Uthman OA (2020) Individual and contextual correlates of mosquito net use among women in Nigeria. Malaria Journal 19(1), 138.

Adewoyin A, Busari O and Aworanti O (2019) Hemoglobin phenotypes in Nigeria: data from a National Reference Laboratory. American Journal of Clinical Pathology 152(Supplement 1), S115-S116.

Ahmed SM and Zerihun A (2010) Possession and usage of insecticidal bed nets among the people of Uganda: is BRAC Uganda health programme pursuing a pro-poor path? PLoS One 5(9), e12660.

Alawode OA, Chima V and Awoleye AF (2019) Household characteristics as determinants of ownership of mosquito nets in urban households in Nigeria. Scientific African 6, e00156.

Ameyaw EK, Adde KS, Dare S and Yaya S (2020) Rural-urban variation in insecticide-treated net utilization among pregnant women: evidence from 2018 Nigeria Demographic and Health Survey. Malaria Journal 19(1), 407.

Amoran OE, Onwumbe OO, Salami OM and Mautin GB (2014) The influence of environmental sanitation on prevalence of malaria in a rural town in south-western Nigeria. Nigerian Journal of Medicine: Journal of the National Association of Resident Doctors of Nigeria 23(3), 254-262.

Andrada A, Herrera S, Inyang U, Mohammed AB, Uhomoibhi P and Yé Y (2019) A subnational profiling analysis reveals regional differences as the main predictor of ITN ownership and use in Nigeria. Malaria Journal 18(1), 185.

Auta A (2012) Demographic factors associated with insecticide treated net use among Nigerian women and children. North American Journal of Medical Sciences 4(1), 40-44.

Babalola OJ, Sambo MN, Idris SH, Ajayi IOO, Ajumobi O and Nguku P (2019) Factors associated with utilization of LLINs among women of child-bearing age in Igabi, Kaduna State, Nigeria. Malaria Journal 18(1), 412.

Barros AJ and Hirakata VN (2003) Alternatives for logistic regression in cross-sectional studies: an empirical comparison of models that directly estimate the prevalence ratio. BMC Medical Research Methodology 3(1), 21.

Britannica (2021) Nigeria - Settlement Patterns. URL: https://www.britannica.com/place/Nigeria (accessed 9th June 2021).

Committee on Educating Health Professionals to Address the Social Determinants of Health, Board on Global Health, Institute of Medicine and National Academies of Sciences, Engineering and Medicine (2016) A Framework for Educating Health Professionals to Address the Social Determinants of Health, Vol. 3. National Academies Press, Washington, DC. URL: https://www.ncbi.nlm.nih.gov/books/NBK395983/ (accessed 30th July 2021).

Dawaki S, Al-Mekhlafi HM, Ithoi I, Ibrahim J, Atroosh WM, Abdulsalam AM et al. (2016) Is Nigeria winning the battle against malaria? Prevalence, risk factors and KAP assessment among Hausa communities in Kano State. Malaria Journal 15(1), 351.

De Silva PM and Marshall JM (2012) Factors contributing to urban malaria transmission in sub-Saharan Africa: a systematic review. Journal of Tropical Medicine 2012, e819563.

Dear NF, Kadangwe C, Mzilahowa T, Bauleni A, Mathanga DP, Duster C et al. (2018) Household-level and surrounding peri-domestic environmental characteristics associated with malaria vectors Anopheles arabiensis and Anopheles funestus along an urban-rural continuum in Blantyre, Malawi. Malaria Journal 17(1), 229.

Deressa W and Ali A (2009) Malaria-related perceptions and practices of women with children under the age of five years in rural Ethiopia. BMC Public Health 9(1), 259.

Diiro GM, Affognon HD, Muriithi BW, Wanja SK, Mbogo C and Mutero C (2016) The role of gender on Malaria preventive behaviour among rural households in Kenya. Malaria Journal 15(1), 1-8

Dike N, Onwujekwe O, Ojukwu J, Ikeme A, Uzochukwu B and Shu E (2006) Influence of education and knowledge on perceptions and practices to control malaria in Southeast Nigeria. Social Science \& Medicine 63(1), $103-106$.

Eteng M, Mitchell S, Garba L, Ana O, Liman M, Cockcroft A and Andersson N (2014) Socio-economic determinants of ownership and use of treated bed nets in Nigeria: results from a cross-sectional study in Cross River and Bauchi States in 2011. Malaria Journal 13(1), 1-8.

Ezire O, Adebayo SB, Idogho O, Bamgboye EA and Nwokolo E (2015) Determinants of use of insecticide-treated nets among pregnant women in Nigeria. International Journal of Women's Health 7, 655-661.

Farid BA (2016) Challenges in the management of malaria in Nigeria: a healthcare system preview. Epidemiology: Open Access 6(3), 1-3.

Federal Ministry of Health (2014) National Malaria Elimination Programme: National Malaria Strategic Plan 2014-2020, Nigeria 2014. URL: https://www.health.gov.ng/doc/Final-NMEP-M_E-Plan-2014-2020-May-3rd-updated-09_05_16.pdf (accessed 22nd January 2021).

Ghahremani L, Faryabi R and Kaveh MH (2014) Effect of health education based on the protection motivation theory on malaria preventive behaviors in rural households of Kerman, Iran. International Journal of Preventive Medicine 5(4), 463-471. 
Giardina F, Kasasa S, Sié A, Utzinger J, Tanner M and Vounatsou P (2014) Effects of vector-control interventions on changes in risk of malaria parasitaemia in sub-Saharan Africa: a spatial and temporal analysis. The Lancet Global Health 2(10), e601-e615.

Gómez KM, Caicedo MA, Gaitán A, Herrera-Varela M, Arce MI, Vallejo AF, Padilla J, Chaparro P et al. (2017) Characterizing the malaria rural-to-urban transmission interface: the importance of reactive case detection. PLoS Neglected Tropical Diseases 11(7), e0005780.

Green EC and Murphy E (2014) Health Belief Model. In American Cancer Society (ed.) The Wiley Blackwell Encyclopedia of Health, Illness, Behavior, and Society, pp. 766-769.

Hahn RA and Truman BI (2015) Education improves public health and promotes health equity. International Journal of Health Services: Planning, Administration, Evaluation 45(4), 657-678.

Hilz R and Wagner M (2018) Marital status, partnership and health behaviour: findings from the German Ageing Survey (DEAS). Comparative Population Studies 43, doi:10.12765/CPoS-2018-08.

Jones CL, Jensen JD, Scherr CL, Brown NR, Christy K and Weaver J (2015) The Health Belief Model as an explanatory framework in communication research: exploring parallel, serial, and moderated mediation. Health Communication 30(6), 566-576.

Jones COH and Williams HA (2004) The social burden of malaria: what are we measuring? The intolerable burden of malaria ii: what's new, what's needed? American Journal of Tropical Medicine and Hygiene 71(2) (Supplement), https://www.ncbi. nlm.nih.gov/books/NBK3746/

Kudom AA and Mensah BA (2010) The potential role of the educational system in addressing the effect of inadequate knowledge of mosquitoes on use of insecticide-treated nets in Ghana. Malaria Journal 9(1), 256.

National Population Commission and ICF (2009) Nigeria Demographic and Health Survey 2008. NPC and ICF, Abuja, Nigeria and Rockville, MD, USA. URL: https://www.dhsprogram.com/publications/publication-fr222-dhs-final-reports.cfm (accessed 30th July 2021).

National Population Commission and ICF (2014) Nigeria Demographic and Health Survey 2013. NPC and ICF, Abuja, Nigeria and Rockville, MD, USA. URL: https:/www.dhsprogram.com/publications/publication-fr293-dhs-final-reports.cfm (accessed 30th July 2021).

National Population Commission and ICF (2019) Nigeria Demographic and Health Survey 2018. NPC and ICF, Abuja, Nigeria and Rockville, MD, USA. URL: https://www.dhsprogram.com/publications/publication-fr359-dhs-final-reports.cfm (accessed 30th July 2021).

Nkoka O, Chipeta MS, Chuang Y-C, Fergus D and Chuang K-Y (2019) A comparative study of the prevalence of and factors associated with insecticide-treated nets usage among children under 5 years of age in households that already own nets in Malawi. Malaria Journal 18(1), 43.

Odongo-Aginya E, Ssegwanyi G, Kategere P and Vuzi PC (2005) Relationship between malaria infection intensity and rainfall pattern in Entebbe peninsula, Uganda. African Health Sciences 5(3), 238-245.

Oduola AO, Olojede JB, Oyewole IO, Otubanjo OA and Awolola TS (2013) Abundance and diversity of Anopheles species (Diptera: Culicidae) associated with malaria transmission in human dwellings in rural and urban communities in Oyo State, Southwestern Nigeria. Parasitology Research 112(10), 3433-3439.

Okunlola OA and Oyeyemi OT (2019) Spatio-temporal analysis of association between incidence of malaria and environmental predictors of malaria transmission in Nigeria. Scientific Reports 9(1), 17500.

Oladimeji KE, Tsoka-Gwegweni JM, Ojewole E and Yunga ST (2019) Knowledge of malaria prevention among pregnant women and non-pregnant mothers of children aged under 5 years in Ibadan, South West Nigeria. Malaria Journal 18, doi:10.1186/s12936-019-2706-1.

Padilla JC, Chaparro PE, Molina K, Arevalo-Herrera M and Herrera S (2015) Is there malaria transmission in urban settings in Colombia? Malaria Journal 14(1), 453.

Pell C, Straus L, Andrew EVW, Meñaca A and Pool R (2011) Social and cultural factors affecting uptake of interventions for malaria in pregnancy in Africa: a systematic review of the qualitative research. PLoS One 6(7), e22452.

Robards J, Evandrou M, Falkingham J and Vlachantoni A (2012) Marital status, health and mortality. Maturitas 73(4), 295-299.

Santos CAS, Fiaccone RL, Oliveira NF, Cunha S, Barreto ML, do Carmo MBB et al. (2008) Estimating adjusted prevalence ratio in clustered cross-sectional epidemiological data. BMC Medical Research Methodology 8(1), 80.

Shimaponda-Mataa NM, Tembo-Mwase E, Gebreslasie M and Mukaratirwa S (2017) Knowledge, attitudes and practices in the control and prevention of malaria in four endemic provinces of Zambia. Southern African Journal of Infectious Diseases 32(1), 29-39.

Singh M, Brown G and Rogerson SJ (2013) Ownership and use of insecticide-treated nets during pregnancy in sub-Saharan Africa: a review. Malaria Journal 12, 268.

Taylor SM, Parobek CM and Fairhurst RM (2012) Impact of haemoglobinopathies on the clinical epidemiology of malaria: a systematic review and meta-analysis. The Lancet Infectious Diseases 12(6), 457-468.

Tobin-West CI and Alex-Hart BA (2011). Insecticide-treated bednet ownership and utilization in Rivers State, Nigeria before a state-wide net distribution campaign. Journal of Vector Borne Diseases 48(3), 133-137. 
Umberson D (1992) Gender, marital status and the social control of health behavior. Social Science \& Medicine 34(8), 907-917.

Van Damme-Ostapowicz K, Krajewska-Kułak E, Rozwadowska E, Nahorski WL and Olszański R (2012) Quality of life and satisfaction with life of malaria patients in context of acceptance of the disease: quantitative studies. Malaria Journal 11, 171.

Watanabe N, Kaneko A, Yamar S, Leodoro H, Taleo G, Tanihata T, Lum JK and Larson PS (2014) Determinants of the use of insecticide-treated bed nets on islands of pre- and post-malaria elimination: an application of the health belief model in Vanuatu. Malaria Journal 13, doi:10.1186/1475-2875-13-441.

World Health Organization (2010) A conceptual framework for action on the social determinants of health. URL: https:// www.who.int/publications-detail-redirect/9789241500852(accessed 20th January 2021).

World Health Organization (2018) WHO World Malaria Report 2017. URL: http://www.who.int/malaria/publications/ world-malaria-report-2017/en/ (accessed 18th January 2021).

World Health Organization (2019a) The E-2020 Initiative of 21 Malaria-Eliminating Countries: 2019 Progress Report. URL: http://www.who.int/malaria/publications/atoz/e-2020-progress-report-2019/en/ (accessed 10th May 2021).

World Health Organization (2019b) World Malaria Report 2019. URL: https://www.who.int/publications-detail-redirect/ 9789241565721 (accessed 18th January 2021).

World Health Organization (2020) Malaria. URL: https://www.who.int/news-room/fact-sheets/detail/malaria (accessed 18th January 2021).

World Health Organization (2021) World Malaria Day: WHO Launches Effort to Stamp Out Malaria in 25 More Countries by 2025. URL: https://www.who.int/news/item/21-04-2021-world-malaria-day-who-launches-effort-to-stamp-out-malariain-25-more-countries-by-2025 (accessed 10th May 2021).

Zou G (2004) A modified Poisson regression approach to prospective studies with binary data. American Journal of Epidemiology 159(7), 702-706.

Zou GY and Donner A (2013) Extension of the modified Poisson regression model to prospective studies with correlated binary data. Statistical Methods in Medical Research 22(6), 661-670.

Cite this article: Duodu PA, Dzomeku VM, Emerole CO, Agbadi P, Arthur-Holmes F, and Nutor JJ (2022). Rural-urban dimensions of the perception of malaria severity and practice of malaria preventive measures: insight from the 2018 Nigeria Demographic and Health Survey. Journal of Biosocial Science 54, 858-875. https://doi.org/10.1017/ S0021932021000420 